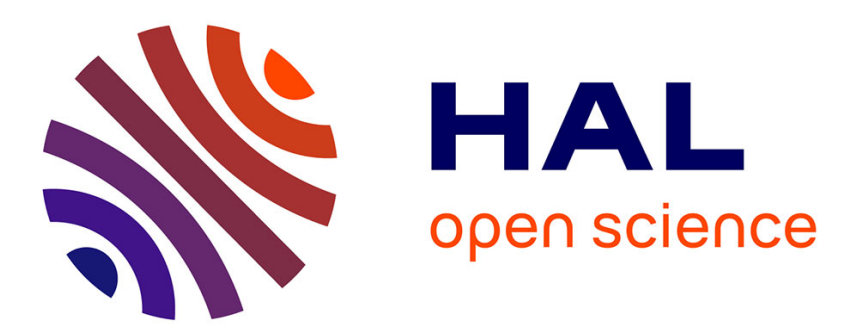

\title{
Note on the determination of the ignition point in forest fires propagation using a control algorithm
}

\author{
Michel Bergmann, Olivier Séro-Guillaume, Sepehr Ramezani
}

\section{To cite this version:}

Michel Bergmann, Olivier Séro-Guillaume, Sepehr Ramezani. Note on the determination of the ignition point in forest fires propagation using a control algorithm. Communications in Numerical Methods in Engineering, 2008, 24, pp.879-896. 10.1002/cnm.990 . inria-00338759

\section{HAL Id: inria-00338759 \\ https://hal.inria.fr/inria-00338759}

Submitted on 14 Nov 2008

HAL is a multi-disciplinary open access archive for the deposit and dissemination of scientific research documents, whether they are published or not. The documents may come from teaching and research institutions in France or abroad, or from public or private research centers.
L'archive ouverte pluridisciplinaire HAL, est destinée au dépôt et à la diffusion de documents scientifiques de niveau recherche, publiés ou non, émanant des établissements d'enseignement et de recherche français ou étrangers, des laboratoires publics ou privés. 


\title{
Note on the determination of the ignition point in forest fires propagation using a control algorithm
}

\author{
M. Bergmann, O. Séro-Guillaume and S. Ramezani
}

November 14, 2006

Laboratoire d'Energétique et de Mécanique Théorique et Appliquée, UMR 7563 CNRS, avenue de la forêt de Haye, BP 160, 54504 Vandoeuvre-lès-Nancy Cedex, France

\begin{abstract}
This paper is devoted to the determination of the origin point in forest fires propagation using a control algorithm. The forest fires propagation are mathematically modelled starting from a reaction diffusion model. A V.O.F. formulation is also used to determine the fraction of the area which is burnt. After having developed the objective functional and its derivative, results from an optimiza-
\end{abstract}


tion process based on the simplex method is presented. It is shown that the ignition point and the final time of the fire propagation are precisely recovered, even for a realistic, non horizontal, terrain.

Key words: Forest fire; reaction diffusion model; optimization

\section{INTRODUCTION}

The simulation of forest fire propagation has several purposes. The prevision of the fire front can help fire fighters to optimize the distribution of fire fighting means, which supposes real time simulation. Another application of simulation is fire prevention. By using terrain data, computer models of propagation can provide information on dangerous areas. The possibility for such models to take into account some aspects of the fire fighting means, such as chemical retardants, is highly desirable as well. In this paper we are concerned by applications dedicated to the analysis of the fire departure. Very often the fire is detected after a while, the exact position and time of the ignition are not known and the determination of the position would be valuable information for fire men who are in charge of the expertise of the fire. The aim of this paper is to address the question of the determination of these initial conditions. The model of propagation, or direct model, considered is a simplified version of the reaction diffusion model proposed in Ref [1]. It is a two dimensional model set on the surface of propagation with a non local heat source term modelling the radiative transfer. The fire front is given by an iso line $T=$ cte of the temperature field. 
We have introduced a Volum Of Fluid (V.O.F.) like formulation using a function which represents the burnt area density. This is a quite different strategy that the one used by Ferragut et al. [2] and Asencio et al. [3] where the fire boundary is obtained by a multivalued operator. The interest of a V.O.F. formulation is that the determination of the critical conditions is formulated as an optimal control problem set with the burnt area density.

The paper is organized as follows: Paragraph 2 is devoted to the presentation of the considered reaction diffusion model, and of the V.O.F. formulation. In paragraph 3 the objective function is introduced and the differential of the functional is computed. The fourth paragraph is devoted to numerical applications.

\section{DIFFUSION REACTION PROPAGATION MO- \\ DELS}

\subsection{General description of the model}

The model considered is deduced from the balance of energy and the balance of mass for the solid fuel:

$(1-\Phi) \rho\left(C_{s}+H_{u} C_{l}\right) \frac{\partial T}{\partial t}=\nabla \cdot(\lambda \nabla T)+h\left(T_{f}-T\right)+(1-\Phi) \rho \frac{\partial H_{u}}{\partial t} L_{e v} \delta_{T=T_{e v}}+M_{r}$

In this relation $T, \rho$ and $\Phi$ represent the temperature, the density of wood, 
and the porosity of the vegetation, $C_{s}$ and $C_{l}$ stand for the heat capacity of the dried wood and of water, $H_{u}$ is the humidity, $T_{f}$ is the temperature of the ambient gas. $M_{r}$ is the radiative flux coming from flames. This model corresponds to a case where the totality of the energy received by the fuel is used for evaporating the water during the process of drying. If the water is free (not inside the vegetal) one has to change the value of the "latent" heat $L_{e v}$. In the zone where pyrolysis occurs, an equation modeling the kinetic of decomposition must be considered:

$$
\frac{\partial \rho}{\partial t}=-\rho f(T)
$$

Indeed the preceding model can be summed up as follows:

(i) In the zone before the evaporation front, denoted by zone $I$, such that:

$$
T<T_{e v} \text { and } \rho>\rho_{e x t},
$$

$$
(1-\Phi) \rho\left(C_{s}+H_{u 0} C_{l}\right) \frac{\partial T}{\partial t}=\nabla \cdot(\lambda \nabla T)+M_{r}+h\left(T_{f}-T\right),
$$

where $C_{s}$ is the heat capacity of the solid constituent of vegetation, $C_{l}$ is the heat capacity of the water, $H_{u 0}$ is the initial humidity; $h$ is the heat loss coefficient, $T_{f}$ is the temperature of the gaseous phase.

(ii) In the evaporation zone, denoted by zone $I I$, such that:

$T=T_{e v}, H_{u}>0$ and $\rho \geq \rho_{e x t}$

$$
-(1-\Phi) \rho L_{e v} \frac{\partial H_{u}}{\partial t}=M_{r}-h\left(T-T_{f}\right) .
$$


$L_{e v}$ is an evaporation latent heat and $\rho_{e x t}$ denotes the extinction density of wood.

(iii) In the intermediary zone between the evaporation zone and the burning zone, denoted by zone $I I I$, such that:

$T_{e v}<T<T_{i}, H_{u}=0$ and $\rho \geq \rho_{e x t}$

$$
(1-\Phi) \rho C_{s} \frac{\partial T}{\partial t}=\nabla \cdot(\lambda \nabla T)+M_{r}-h\left(T-T_{f}\right)
$$

$T_{i}$ is the ignition temperature.

(iv) In the burning zone, denoted by zone $I V$, such that:

$T \geq T_{i}, H_{u}=0$ and $\rho \geq \rho_{\text {ext }}$

$$
(1-\Phi) \rho C_{s} \frac{\partial T}{\partial t}=\nabla \cdot(\lambda \nabla T)+M_{r}-h\left(T-T_{f}\right)
$$

The variation of mass due to chemical reactions is:

$$
\frac{\partial \rho}{\partial t}=-\rho A \exp (-E / R T)
$$

where $A$ is a constant and $E$ is the activation energy of the pyrolysis.

(v) the burnt zone, denoted by zone $\mathrm{V}$, such that:

$\rho=\rho_{\text {ext }}$

$$
(1-\Phi) \rho C_{s} \frac{\partial T}{\partial t}=\nabla \cdot(\lambda \nabla T) M_{r}-h\left(T-T_{f}\right) .
$$


All the preceding described regions are illustrated in the following figure 1:

\section{$2.2 \quad$ Flame model}

The vegetation is supposed to be thin and set on a plane $S_{f}$. The flame is supposed to be at constant known temperature $T_{f}$ and each flame element is supposed to be directed by a unit vector $\boldsymbol{F}$ parallel to the velocity of the gas $\boldsymbol{V}_{f}$, the emitting point is denoted by $P$ and the receiving point by $M, O$ is the flame foot (cf. Figure 2).

The global unit vectors of the global co-ordinates system are denoted by $\left(\boldsymbol{e}_{1}, \boldsymbol{e}_{2}, \boldsymbol{e}_{3}\right), \boldsymbol{e}_{3}$ being the vertical direction. The vector $\boldsymbol{n}$ is the unit normal to the upper plane, (i.e. unit normal to the receiving surface) of the vegetation at point $M$. The angle between $\boldsymbol{F}$ and the vertical is denoted by $\alpha_{f}=\left(\boldsymbol{e}_{3}, \boldsymbol{F}\right)$. The flame elements are supposed to have a length $l_{f}$. If the fire front is supposed to be thin, one can show, that the radiative heat flux is given by convolution integral calculated on the burning zone denoted by $S_{f}$ :

$$
\begin{aligned}
M_{r} & =\int_{S_{f}} \phi(\boldsymbol{y}) G(\boldsymbol{x}-\boldsymbol{y}) d y_{1} d y_{2} \\
& =K_{f} \frac{B T^{4}}{\pi} \int_{S_{f}} \frac{\left(\boldsymbol{F}\left(1-\cos \theta_{f}\right)-\boldsymbol{w}\left(\cos \beta-\cos \left(\beta+\theta_{f}\right)\right)\right) \cdot \boldsymbol{n}}{r \sin ^{2} \beta} d x_{1} d x_{2},
\end{aligned}
$$

with, cf. appendix A for notation and derivation, $r$ being the distance between the emitting and the receiving points, and $K_{f}$ the absorption coefficient of the flame.

The velocity of the gas is the sum of the vertical velocity of the gases and of the wind 


$$
\boldsymbol{V}_{f}=\boldsymbol{v}_{g}+\boldsymbol{V}
$$

where $\boldsymbol{v}_{g}=\sqrt{g h_{f}} \boldsymbol{e}_{3}$ is the vertical flame gas velocity, $h_{f}$ being the flame height and $\boldsymbol{V}$ is the wind velocity.

\section{$2.3 \quad$ V.O.F. FORMULATION}

In fact the propagation of fire in the preceding modeling is a free boundary problem. Let us derive a V.O.F. formulation [4]. For sake of simplicity let us assume that there is no humidity that is $H_{u}=0$. Let $\chi(\boldsymbol{x}, t)$ the characteristic function of the burning zone, i.e. such that $\chi(\boldsymbol{x}, t)=1$ if the point $\boldsymbol{x}$ lies in the burning zone at time $t$ and $\chi(\boldsymbol{x}, t)=0$ elsewhere. The characteristic function, considered as a distribution, satisfies the equation:

$$
\frac{\partial \chi}{\partial t}+\nabla \chi \cdot \boldsymbol{w}=0
$$

In relation (11) $\boldsymbol{w}$ is the normal velocity of the fire front, it is equal to:

$$
\boldsymbol{w}=-\left.\frac{\frac{\partial T}{\partial t}}{|\boldsymbol{\nabla} T|^{2}} \boldsymbol{\nabla} T\right|_{T=T_{i}}
$$

We can consider now a mollifier $m_{h}(\boldsymbol{x})$, i.e. a function such that $m_{h}(\boldsymbol{x})>0$, $\int_{\mathbb{R}^{2}} m_{h}(\boldsymbol{x}) d \boldsymbol{x}=1$ and the function tends to a Dirac distribution when $h \rightarrow 0$. This function can be chosen as smooth as desired, so that the function defined by the convolution: 


$$
\alpha(\boldsymbol{x}, t)=\left(\chi * m_{h}\right)(\boldsymbol{x}, t)=\int_{\mathbb{R}^{2}} \chi(\boldsymbol{y}, t) m_{h}(\boldsymbol{x}-\boldsymbol{y}) d \boldsymbol{y}
$$

is a regular approximation of the characteristic function $\chi$. Now an approximation of the system of propagation is:

$$
\begin{gathered}
(1-\Phi) \rho C_{s} \frac{\partial T}{\partial t}=\nabla \cdot(\lambda(\alpha) \nabla T)+M_{r}(\alpha)-h\left(T-T_{f}\right) \\
\frac{\partial \alpha}{\partial t}+\nabla \alpha \cdot \boldsymbol{w}=0 \\
\frac{\partial \rho}{\partial t}=-\rho A \exp (-E / R T)
\end{gathered}
$$

and now the radiative heat is given by:

$$
M_{r}=\int_{\mathbb{R}^{2}} \alpha(\boldsymbol{y}, t) \phi(\boldsymbol{y}) G(\boldsymbol{x}-\boldsymbol{y}) d y_{1} d y_{2}
$$

The velocity $\boldsymbol{w}$ is an extension of the front velocity given by:

$$
\boldsymbol{w}=-\frac{\frac{\partial T}{\partial t}}{|\boldsymbol{\nabla} T|^{2}} \nabla T
$$

The system (13)-(18) is now set on the whole plane $\mathbb{R}^{2}$. The study of the numerical algorithm for solving this system is not addressed in this paper. 


\section{The control algorithm for the determination of the ignition point}

\subsection{Definition of the objective function and computation of its differential}

Let us consider now at time $t_{f}$ a burnt area whose characteristic function is denoted by $\chi_{f}$, to which it corresponds by convolution a function $\alpha_{f}$. In fact we are interested in finding the position $\left(x_{0}, y_{0}\right)$ and the time $t_{0}$ of ignition. Let us add to the system (14)-(18) the initial conditions

$$
\begin{gathered}
\chi\left(x, y, t_{0}\right)=\delta_{x=x_{0}, y=y_{0}} \\
T\left(x, y, t_{0}\right)=T_{i} \delta_{x=x_{0}, y=y_{0}},
\end{gathered}
$$

Or the related relations written with the regularized function $\alpha$.

It is now natural to set the problem as an optimal control problem.

Find $\left(x_{0}, y_{0}, t_{0}\right)$ such that $\mathcal{J}=\int_{\mathbb{R}^{2}}\left|\chi\left(x, y, t_{f}\right)-\chi_{f}(x, y)\right|^{2} d x d y$ is minimum.

The numerical calculation of the integral $\mathcal{J}$ in the problem $(21)$ will be replaced by the approximation:

$$
\mathcal{J}_{a}=\int_{\mathbb{R}^{2}}\left|\alpha\left(x, y, t_{f}\right)-\alpha_{f}(x, y)\right|^{2} d x d y
$$


In fact $\mathcal{J}$ is an integral calculated on the domain $\Omega-\Omega_{f}, \Omega$ being the domain whose characteristic function is $\chi$ and can be written:

$$
\mathcal{J}=\int_{\Omega-\Omega_{f}} d x d y
$$

In practice, the constrained optimization problem (21) can be solved using two different class of algorithms. The first class is composed by the stochastic algorithms like the genetic ones. These kinds of algorithms permits to find the global optimizer of an objective function but for a prohibitive computational time in engineering applications. The second class, composed mainly by the gradient-based methods, seems to be more efficient but does not guaranteed to converge towards the global optimizer. Under theses remarks it is thus clear that a balance between the computational time and the performance of the solution has to be made. In order to reduce the computational costs involved during stochastic process Armijanov [5] has developed a new class of genetic algorithm where the range of the exploration region is re-adapted for each generation. Stochastic procedures are also often used with interpolation methods [6, 7]. In this paper we choose to solve the constrained optimization problem (21) with two deterministic methods. The first one is the Polack-Ribière conjugate gradient algorithm, which requires the determination of the differential of $\mathcal{J}$, coupling with a backtracking Armijo line search. The second one is the non linear Nelder Mead simplex method [8] which does not require the differentiability of the objective functional. Both algorithms have been used numerically, and for sake of completeness we give the differential of the objective functional with respect to 
$x_{0}$, analog results would be obtained for the other components. The differential of $\mathcal{J}$ can be written:

$$
\frac{\partial \mathcal{J}}{\partial x_{0}}=\int_{\mathbb{R}^{2}} 2\left(\chi\left(x, y, t_{f}\right)-\chi_{f}(x, y)\right) \frac{\partial \chi}{\partial x_{0}} d x d y
$$

The term $\frac{\partial \chi}{\partial x_{0}}$ being solution of the system of equations:

$(1-\Phi)\left(\rho C_{s} \frac{\partial T^{\prime}}{\partial t}+\rho^{\prime} C_{s} \frac{\partial T}{\partial t}\right)=\nabla \cdot\left(\lambda\left(\chi^{\prime}\right) \nabla T\right)+\nabla \cdot\left(\lambda(\chi) \nabla T^{\prime}\right)+M_{r}\left(\chi^{\prime}\right)-h T^{\prime}$

$$
\frac{\partial \chi^{\prime}}{\partial t}+\nabla \chi^{\prime} \cdot \boldsymbol{w}+\nabla \chi \cdot \boldsymbol{w}^{\prime}=0
$$

$\frac{\partial \rho^{\prime}}{\partial t}=-\chi^{\prime} \rho A \exp (-E / R T)-\chi \rho^{\prime} A \exp (-E / R T)-\chi \rho \frac{E T^{\prime}}{R T^{2}} A \exp (-E / R T)$,

$$
M_{r}=\int_{\mathbb{R}^{2}} \chi^{\prime}(\boldsymbol{y}, t) \phi(\boldsymbol{y}) G(\boldsymbol{x}-\boldsymbol{y}) d y_{1} d y_{2}
$$

The notation $T^{\prime}$ stands for $\frac{\partial T}{\partial x_{0}}$, and the initial conditions are:

$$
\begin{gathered}
T^{\prime}\left(x, y, t_{0}\right)=T_{i} \delta_{x=x_{0}, y=y_{0}}^{\prime} \\
\chi^{\prime}\left(x, y, t_{0}\right)=\delta_{x=x_{0}, y=y_{0}}^{\prime}
\end{gathered}
$$

The distribution $\delta_{x=x_{0}, y=y_{0}}^{\prime}$ is defined by: 


$$
\left\langle\varphi, \delta_{x=x_{0}, y=y_{0}}^{\prime}\right\rangle=-\frac{\partial \varphi}{\partial x}\left(x_{0}, y_{0}\right)
$$

for any function with compact support in $\mathbb{R}^{2}$.

In fact the terminal position of the fire front will never be known exactly, we will consider perturbations $\delta \chi_{f}$ of the boundary. Let us consider virtual motion:

$$
\boldsymbol{x} \mapsto \boldsymbol{x}+\varepsilon \boldsymbol{\tau}(\boldsymbol{x})
$$

in such a way that every points $\boldsymbol{x}$ is moved up to first order in $\varepsilon$. Let us defined the translated characteristic function:

$$
\bar{\chi}_{f}(\varepsilon)=\chi_{f}(\boldsymbol{x}+\varepsilon \boldsymbol{\tau}(\boldsymbol{x})) .
$$

Then the variations of the characteristic function are defined by:

$$
\delta \chi_{f}=\varepsilon \frac{\partial \bar{\chi}_{f}}{\partial \varepsilon}(\varepsilon=0)=\varepsilon \nabla \chi_{f} \cdot \boldsymbol{\tau}=-\varepsilon \delta_{\partial \Omega_{f}} \boldsymbol{n} \cdot \boldsymbol{\tau} .
$$

In relation (34), $\delta_{\partial \Omega_{f}}$ stands for the Dirac distribution on $\partial \Omega_{f}, \boldsymbol{n}$ is the unit outward normal. Then we can compute the variation, or sensitivity of the objective functional:

$$
\delta \mathcal{J}=\mathcal{J}\left(\chi_{f}+\delta \chi_{f}\right)-\mathcal{J}\left(\chi_{f}\right)=-2 \int_{\Omega_{f}}\left(\chi^{-} \chi_{f}\right) \delta \chi_{f} d x d y+\int_{\Omega_{f}}\left(\delta \chi_{f}\right)^{2} d x d y
$$

The computation of $\delta \mathcal{J}$ must be done for $\chi=\chi_{f}$ then:

$$
\delta \mathcal{J}=\int_{\Omega_{f}}\left(\delta \chi_{f}\right)^{2} d x d y=\varepsilon^{2} \int_{\partial \Omega_{f}} \tau^{2} d s
$$


where $d s$ denotes the curvilinear coordinate along the line boundary $\partial \Omega_{f}$.

In equation (36) we have set $\tau=\boldsymbol{\tau} \cdot \boldsymbol{n}$. As a function defined on the boundary $\partial \Omega_{f}$ of the final domain, $\tau$ is a periodic function of the arc length $s$ and can be developed in Fourier series:

$$
\tau=\sum_{n} \tau_{n} \exp (-2 i \pi n s / L),
$$

where $L$ is the total length of $\partial \Omega_{f}$. With these notations, the sensitivity of the functional is:

$$
\delta \mathcal{J}=\varepsilon^{2} \sum_{n} \tau_{n}^{2}
$$

\section{Some computational application}

In order to test the algorithm we have first considered a propagation on a horizontal terrain with a uniform density of vegetation, the parameters of the propagation model are the one considered in table 1 . Note that we have not taken into account for this simulation the diffusivity term $\nabla \cdot(\lambda \nabla T)$ in equations (1) (and in the other ones derived from this one) because the value of the parameter $\lambda$ is not physically yet well defined.

The ignition point is arbitrary chosen such that $x_{0}=y_{0}=1000 \mathrm{~m}$. After $t_{f}=120 m n$ of propagation the fire front being a circle has been perturbed by a sinus, that is 
Table 1: Parameters values of the propagation model.

$$
\begin{array}{cc}
C_{s}=2400 \mathrm{~J} \cdot \mathrm{kg}^{-1} \cdot \mathrm{K}^{-1} & C_{l}=4180 \mathrm{~J} \cdot \mathrm{kg}^{-1} \cdot \mathrm{K}^{-1} \\
\delta=1 \mathrm{~m} & K_{f}=0.2 \mathrm{~m}^{-1} \\
h=20 \mathrm{~J} \cdot \mathrm{m}^{-2} \cdot \mathrm{s}^{-1} \cdot K^{-1} & L_{e v}=2.250 \times 10^{6} \mathrm{~J}_{\mathrm{kg}} \mathrm{kg}^{-1} \\
T a=300 \mathrm{~K} & T_{e v}=373 \mathrm{~K} \\
h_{f} \simeq 2 \mathrm{~m} & T_{f}=1200 \mathrm{~K} \\
& \\
\delta \chi_{f}=-\varepsilon \delta_{\partial \Omega_{f}} \boldsymbol{n} \cdot \boldsymbol{\tau}=a \sin \left(\omega s \delta_{\partial \Omega_{f}}\right) .
\end{array}
$$

So that the equation for the new boundary is

$$
\rho=r+a \sin (2 \pi n r \theta / 2 \pi r)=r+a \sin (n \theta)
$$

in polar co-ordinate. This new curve is considered as the noisy perturbated final fire front, see figure 3 .

As mentioned before, two optimization algorithms have been used, PolackRibière conjugate gradient and Nelder Mead simplex, see ref [8]. The most efficient has revealed to be the simplex and the results described here are the one obtained by this way.

Figure 5 represents the evolution of the calculated at each iteration fire ignition point position versus the optimization iterations numbers. It is noticeable that after thirty iterations, the value of the objective function stops to decrease significantly (see figure 4), the real ignition point, i.e. $x_{0}=1000 \mathrm{~m}$ and $y_{0}=1000 \mathrm{~m}$, is precisely recovered. 
After the same optimization iterations numbers, the final propagation time $t_{f}=120 m n$ is also precisely recovered as we can see in figure 6 .

In order to test the efficiency of this study we have secondly taken under consideration a much more complicated case. We have thus considered a propagation on a non horizontal terrain, with a uniform density of vegetation and with the same parameters as in the previous case. The topology of the terrain under consideration is composed by two distinct bumps as one can show in figure 8 . The $z$-coordinates of the terrain is given by

$z(x, y)=z_{0}+h_{b 1} \exp \frac{-\left(x-x_{b 1}\right)^{2}}{A} \exp \frac{-\left(y-y_{b 1}\right)^{2}}{A}+h_{b 2} \exp \frac{-\left(x-x_{b 2}\right)^{2}}{A} \exp \frac{-\left(y-y_{b 2}\right)^{2}}{A}$

where $z_{0}=1 \mathrm{~m}$, the coordinate of the center of the first and second bumps are $\left(x_{b 1}, y_{b 1}\right)=(1010 m, 1030 m)$ and $\left(x_{b 2}, y_{b 2}\right)=(970 m, 970 m)$ respectively, the relative maximal height of the first and second bumps are $h_{b 1}=3 \mathrm{~m}$ and $h_{b 2}=2 m$ respectively, and the value of the smooth factor surface $A$ is $1000 \mathrm{~m}^{2}$.

The ignition point is once again arbitrary taken to be $x_{0}=y_{0}=1000 \mathrm{~m}$. After $t_{f}=120 \mathrm{mn}$ of propagation the fire front, which is not a circle now, has been perturbed by a sinus too (see figure 9 ).

Once again, after thirty iterations the value of the objective functional tends to zero (see figure 10). The ignition point $x_{0}=y_{0}=1000 \mathrm{~m}$ and the final time $t_{f}=120 \mathrm{mn}$ are also precisely recovered (see figures 11 and 12 respectively).

The sensitivities of the objective function is now studied. Several numerical optimizations have been done for different values of the parameters $a$ located between 1 and 10 by steps equal to 1 , for a fixed value $n=20$, see equation (40) 
for the signification of $a$ and $n$. Each numerical optimization gave rise to an optimal value $\mathcal{J}_{\min }$ of the objective function which is nothing else that $\delta \mathcal{J}$ because $\mathcal{J}\left(\chi_{f}\right)=0$. These values are plotted in figure 7 . It is noticeable as foreseen in relations (37) and (38) that the optimal value $\mathcal{J}_{\min }$ vary approximatively as the square of the parameters $a$. Indeed, the more the fire front is perturbated and the more it is difficult to recovered the ignition point.

This simple numerical experiments show that it is possible to recover the initial point and time of the departure of the fire, in an deterministic way, using an optimal control formulation and a simplex optimisation algorithm. However, the question on the existence and uniqueness of the optimal solution of such an optimization problem is still an open question. Numerically, it has to be precise that the same solutions are obtained in a stochastic way using a standard genetic algorithm. It is shown in practice that this kind of algorithms almost give the optimal solution, but for high numerical costs.

\section{Conclusion}

This paper is dedicated in a first approach to the finding of the ignition point in forest fires propagation. The problem as been set as an optimal control problem. The diffusion has not taken into account for a sake of simplicity and because diffusivity has been obtained after an homogenization process and its value is not clearly determined. This will be the purpose of an other paper. The objective functional and its derivatives versus each control parameters were derived. 
This gradient has been used in a conjugate gradient optimization method, but the simplest and effective optimization method is the simplex one. Using this optimization method the ignition point and the final time of forest fires propagation were precisely recovered even for a realistic non horizontal terrain where only noisy measurement of the fire line is available. This will be extended to a diffusive model and with a fire front randomly perturbated. These results could be very helpful for firemen who are in charge of an expertise of the fire.

\section{Acknowledgments}

This research has been done under the support of the European community, research contract "PREVIEW" n EVG1-CT-2001-00043.

\section{References}

[1] Margerit J, Séro-Guillamue O. Modelling Forest Fires. Part II: Reduction to Two-Dimensional Models and Simulation of Propagation International Journal of Heat and Mass Transfer, 2002; 45(8):1723-1737.

[2] Ferragut L., Asensio M.I., Monedero S. Modelling Radiation and Moisture Content in Fire Spread Commun. Numer. Meth. Engng., 2006; (In press).

[3] Asensio MI., Ferragut L., Simon J. A convection model for fire spread simulation Appl. Math. Letter, 2005; 6:673-677. 
[4] Hirt CW, Nichols BD. Volume of Fluid (VOF) method for the Dynamics of Free Boundaries Journal of Computational Physics, 1981; 39:201-225.

[5] Amirjanov A. A changing range genetic algorithm Int. J. Numer. Meth. Engng., 2004; 61:2660-2674.

[6] Alotto P., Nervi MA. An efficient hybrid algorithm for the optimization of problems with several local minima Int. J. Numer. Meth. Engng., 2001; 50:847-868.

[7] Li C., Priemer R, Cheng KH. Optimization by random search with bumps Int. J. Numer. Meth. Engng., 2004; 60:1301-1315.

[8] Nocedal J, Wright SJ. Numerical Optimization Sprinfer series in operation research, 1999. 


\section{A Derivation of the relation (9)}

Let us now derive relation (9).

We will assume that the flame and the vegetation are grey medium with constant absorption coefficients $K_{f}, K_{v}$. Then if the temperature $T_{f}$ of the flame is constant the integration of the radiative transfer equation gives for the intensity

$$
i(s)=\frac{B T_{f}^{4}}{\pi}\left(1-e^{-K_{f}\left(s_{2}-s_{1}\right)}\right) e^{-K_{v}\left(s-s_{3}\right)}+K_{v} \int_{s_{3}}^{s} i_{b}(\bar{s}) e^{-K_{v}(s-\bar{s})} d \bar{s} .
$$

If the flame is assumed thin $\left(1-e^{-K_{f}\left(s_{2}-s_{1}\right)}\right) \simeq K_{f}\left(s_{2}-s_{1}\right)=K_{f} \int_{s_{1}}^{s_{2}} d \bar{s}$ and the preceding relation becomes:

$$
i(s)=K_{f} \frac{B T_{f}^{4}}{\pi} e^{-K_{v}\left(s-s_{3}\right)} \int_{s_{1}}^{s_{2}} d \bar{s}+K_{v} \int_{s_{3}}^{s} i_{b}(\bar{s}) e^{-K_{v}(s-\bar{s})} d \bar{s} .
$$

Then the radiative flux density received by a surface with normal $\boldsymbol{n}_{i}$ is:

$\boldsymbol{q}_{r}(M) \cdot \boldsymbol{n}_{i}=K_{f} \frac{B T_{f}^{4}}{\pi} \int_{\Omega_{f}} \frac{e^{-K_{v} \boldsymbol{a M}}}{\boldsymbol{P} \boldsymbol{M}^{\mathbf{2}}} \boldsymbol{u} \cdot \boldsymbol{n}_{i} d \Omega(P)+K_{v} \frac{B T_{v}^{4}}{\pi} \int_{\Omega_{v}} \frac{e^{-K_{v} \boldsymbol{A M}}}{\boldsymbol{A M}^{\mathbf{2}}} \boldsymbol{u} \cdot \boldsymbol{n}_{i} d \Omega(P)$

Where $\Omega_{f}$ is the domain occupied by the flame and $\Omega_{v}$ is the domain occupied by the vegetation. In the limit $\delta \rightarrow 0$ the right hand side of (42) reduces to:

$$
\boldsymbol{q}_{r}(M) \cdot \boldsymbol{n}=K_{f} \frac{B T_{f}^{4}}{\pi} \int_{\Omega_{f}} \frac{1}{\boldsymbol{P} \boldsymbol{M}^{\mathbf{2}}} \boldsymbol{u} \cdot \boldsymbol{n} d \Omega(P)
$$

This triple integral can be reduced to a double integral. Let us consider that each element of flames is directed by a unit vector $\boldsymbol{F}, \boldsymbol{n}$ is the unit vector 
normal to the plane $\Pi_{v}$ which is the top of vegetation at the receiving point $M, f$ is the unit vector representing the direction of the orthogonal projection of the flame $\boldsymbol{F}$ on the plane, see figure 2.

If we consider an absolute co-ordinate system $\left(O, \boldsymbol{e}_{1}, \boldsymbol{e}_{2}, \boldsymbol{e}_{3}\right)$ (not drawn on figure above), we define the following angles:

$$
\left(\boldsymbol{e}_{3}, \boldsymbol{F}\right)=\alpha_{f}, \quad(\boldsymbol{F}, \boldsymbol{O} \boldsymbol{M})=\beta .
$$

The emitting point on the flame is the point $P$, the point $O$ is the flame foot , and we consider the radial co-ordinate $r$, such that:

$$
\|\boldsymbol{P} \boldsymbol{M}\|=\rho, \quad \boldsymbol{O P}=\xi \boldsymbol{F}, \quad \text { and } \quad \boldsymbol{O} \boldsymbol{M}=r \boldsymbol{w} .
$$

Then $\boldsymbol{P} \boldsymbol{M}=\boldsymbol{O} \boldsymbol{M}-\boldsymbol{O P}=r \boldsymbol{w}-\xi \boldsymbol{F}$, and $\boldsymbol{u} \cdot \boldsymbol{n}=\frac{1}{\rho}(r \boldsymbol{w} \cdot \boldsymbol{n}-\xi \boldsymbol{F} \cdot \boldsymbol{n})$.

In the triple integral of the right hand side of (44) we integrate first along the flame, with the previous notations, we obtain:

$$
M_{r}=-\boldsymbol{q}_{r}(M) \cdot \boldsymbol{n}=-K_{f} \frac{B T_{f}^{4}}{\pi} \int_{S_{f}} \frac{d x d y}{\cos \alpha_{f}} \int_{0}^{l_{f}} \frac{1}{\rho^{3}}(r \boldsymbol{w} \cdot \boldsymbol{n}-\xi \boldsymbol{F} \cdot \boldsymbol{n}) d \xi
$$

In (47) $S_{f}$ is the burning surface and $l_{f}$ the local flame length. The simple integrals $\int_{0}^{l_{f}} \frac{\xi}{\rho^{3}} d \xi$ and $\int_{0}^{l_{f}} \frac{r}{\rho^{3}} d \xi$ can be evaluated. Let us consider the triangle $O P M$ and the different angles in this triangle $c f$. figure below.

Then we have the following relations:

$$
\frac{\rho}{\sin \beta}=\frac{r}{\sin \alpha}=\frac{\xi}{\sin \theta} \quad \text { with } \quad \alpha+\beta+\theta=\pi .
$$


Then $\theta$ can be used as parameter. The derivation of (48) gives:

$$
d \xi=r \frac{\sin \beta}{\sin ^{2}(\beta+\theta)} d \theta=r \frac{\sin \beta}{\sin ^{2} \alpha} d \theta
$$

Then:

$$
\begin{gathered}
I_{1}=\int_{0}^{l_{f}} \frac{r}{\rho^{3}} d \xi=\frac{1}{r \sin ^{2} \beta} \int_{0}^{\theta_{f}} \sin (\beta+\theta) d \theta=\frac{1}{r \sin ^{2} \beta}\left(\cos \beta-\cos \left(\beta+\theta_{f_{m}}\right)\right) \\
I_{2}=\int_{0}^{l_{f}} \frac{\xi}{\rho^{3}} d \xi=\frac{1}{r \sin ^{2} \beta} \int_{0}^{\theta_{f}} \sin \theta d \theta=\frac{1}{r \sin ^{2} \beta}\left(1-\cos \theta_{f_{m}}\right)
\end{gathered}
$$

Once (50) and (51) are put in the integral one obtains:

$$
M_{r}=K_{f} \frac{B T_{f}^{4}}{\pi} \int_{S_{f}} \frac{\left\{\boldsymbol{F}\left(1-\cos \theta_{f_{m}}\right)-\boldsymbol{w}\left(\cos \beta-\cos \left(\beta+\theta_{f_{m}}\right)\right)\right\} \cdot \boldsymbol{n}}{r \sin ^{2} \beta} d x d y .
$$

With $\theta_{f_{m}}$ and $\beta$ solutions to the equations:

$$
\cos \beta=\boldsymbol{F} \cdot \frac{\boldsymbol{O M}}{r}, \quad \text { and } \quad \cot \theta_{f_{m}}=\frac{r}{l_{f} \sin \beta}-\cot \beta
$$

\section{B Description of the optimal control algorithm}

We consider the following optimisation problem:

$$
\min _{\boldsymbol{x}} \mathcal{J}(\boldsymbol{x})
$$

with $\mathcal{J}: \mathbb{R}^{n} \mapsto \mathbb{R}$ is a continuous function. The gradient of the objective function $\mathcal{J}$ according to some control parameters $\boldsymbol{x}$ is noted $\boldsymbol{\nabla} \mathcal{J}$. 
As it was mentioned in $\S 3.1$, we use the Polack-Ribière conjugate gradient algorithm coupling with a backtracking Armijo line search.

\section{B.1 Polack-Ribière conjugate gradient algorithm}

Choose an initial iterate $\boldsymbol{x}_{0}$. Calculus of $\mathcal{J}_{0}=\mathcal{J}\left(\boldsymbol{x}_{0}\right)$ and $\boldsymbol{\nabla} \mathcal{J}_{0}=\boldsymbol{\nabla} \mathcal{J}\left(\boldsymbol{x}_{0}\right)$. The algorithm is initialized by a steepest descent step, $\boldsymbol{d}_{0}=-\nabla \mathcal{J}_{0}$.

While a stopping criterium is not satisfied, do:

1. Determination of a step $\alpha_{k}$ (see $\S$ B.2). Calculus of a new iterate $\boldsymbol{x}_{k+1}=$ $\boldsymbol{x}_{k}+\alpha_{k} \boldsymbol{d}_{k}$

2. Evaluation of a new gradient $\nabla \mathcal{J}_{k+1}$;

3. Construction of a new direction of descent $\boldsymbol{d}_{k+1}=-\nabla \mathcal{J}_{k+1}+\beta_{k+1} \boldsymbol{d}_{k}$, with $\beta_{k+1}=\frac{\nabla \mathcal{J}_{k+1}^{T}\left(\nabla \mathcal{J}_{k+1}-\nabla \mathcal{J}_{k}\right)}{\nabla \mathcal{J}_{k}^{T} \nabla \mathcal{J}_{k}}$

4. $\mathrm{k}=\mathrm{k}+1$

\section{B.2 Backtracking Armijo line search}

The Armijo condition writes:

$$
\mathcal{J}\left(\boldsymbol{x}_{k}+\alpha_{k} \boldsymbol{d}_{k}\right) \leq \mathcal{J}\left(\boldsymbol{x}_{k}\right)+\omega_{1} \alpha_{k}\left\langle\nabla \mathcal{J}_{k}, \boldsymbol{d}_{k}\right\rangle
$$

where $\omega_{1}$ is a small parameters (usually $\omega_{1}=10^{-4}$ ).

Initialisations: choose a step $\alpha_{k}^{1}>0$ and a parameter $\left.\tau \in\right] 0,1[. i=1$.

1. Test: the step $\alpha_{k}^{i}$ is accepted if it verifies the Armijo relation (55) :

$$
\mathcal{J}\left(\boldsymbol{x}_{k}+\alpha_{k}^{i} \boldsymbol{d}_{k}\right) \leq \mathcal{J}\left(\boldsymbol{x}_{k}\right)+\omega_{1} \alpha_{k}^{i}\left\langle\nabla \mathcal{J}_{k}, \boldsymbol{d}_{k}\right\rangle
$$


If not:

2. Choose $\alpha_{k}^{i+1} \in\left[\tau \alpha_{k}^{i},(1-\tau) \alpha_{k}^{i}\right]$

3. $i=i+1$ and $\alpha_{k}=\alpha_{k}^{i}$. Return to 1 .

The parameter $\tau$ is usually taken to be equal to $10^{-2}$.

Usually, only 2 or 3 evaluations of the objective function are necessary for one main step of this optimal control algorithm, composed by the determination of the direction of descent $\boldsymbol{d}$ plus the line search (determination of the step $\alpha$ ).

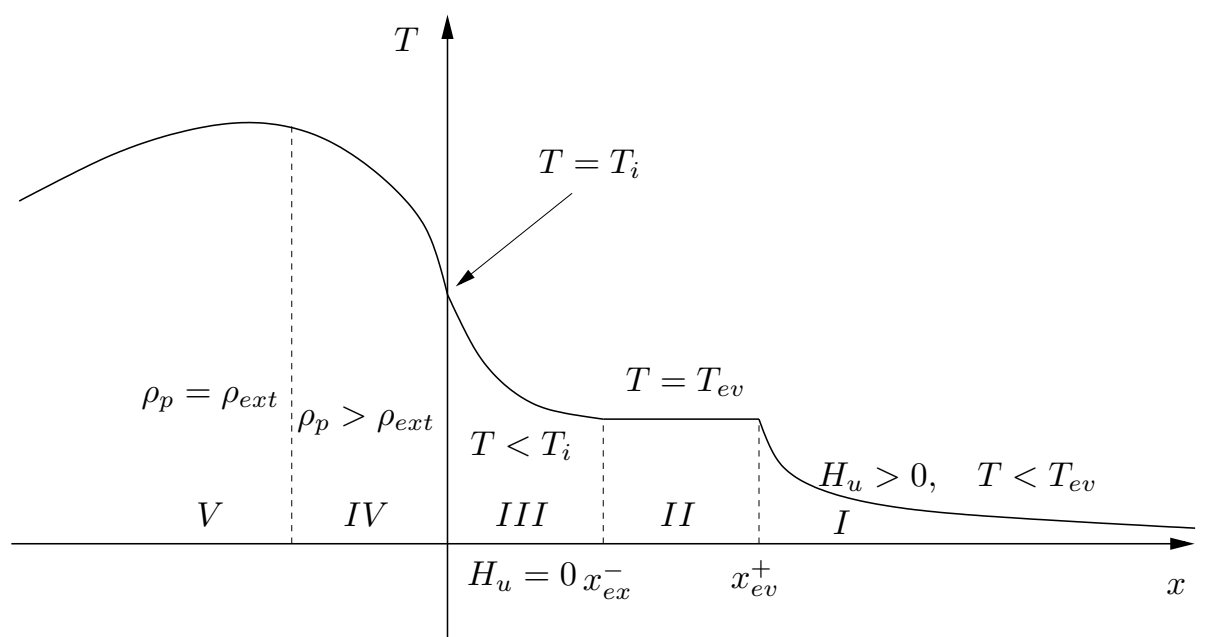

Figure 1: Different zones related to the spreading in a one dimensional propagation, the evaporation zone is the interval $] x_{e v}^{-}, x_{e v}^{+}[$. 


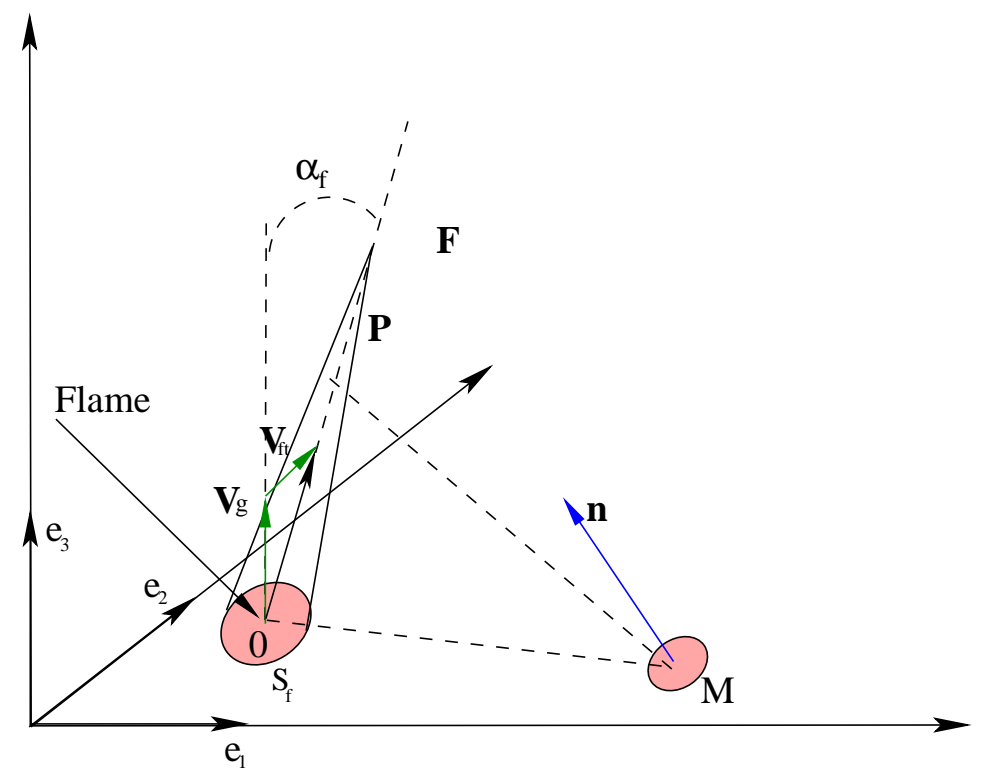

Figure 2: Radiation of the flame. 


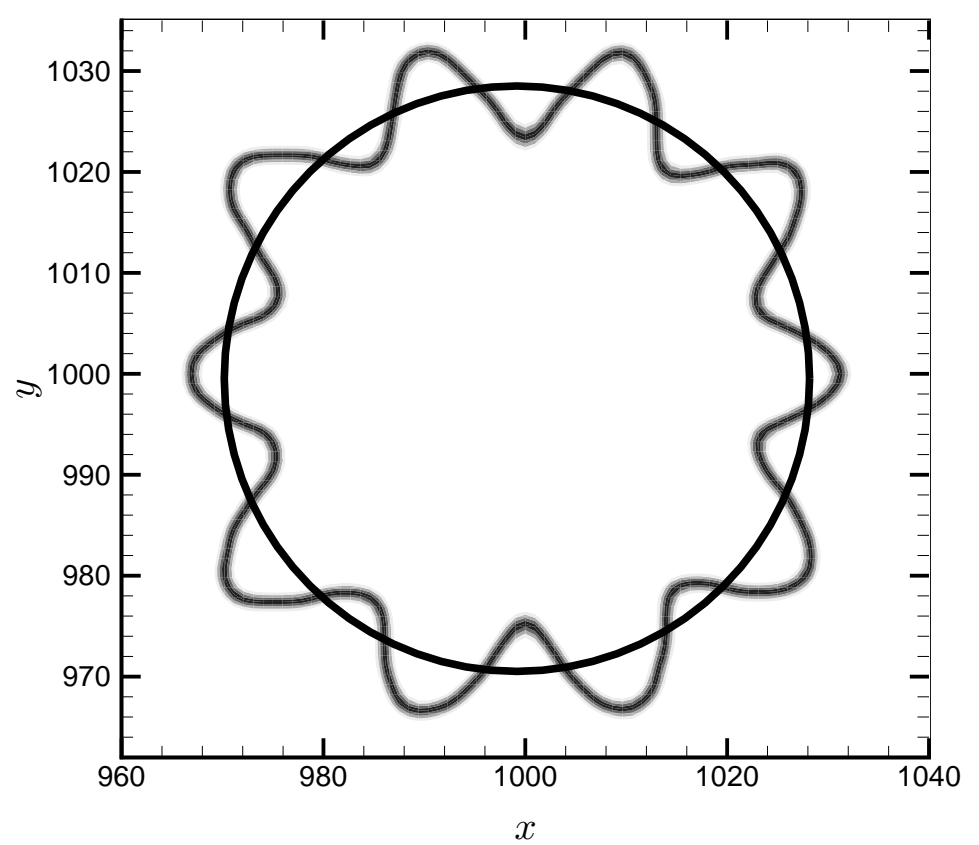

Figure 3: Real and perturbated fire front. 


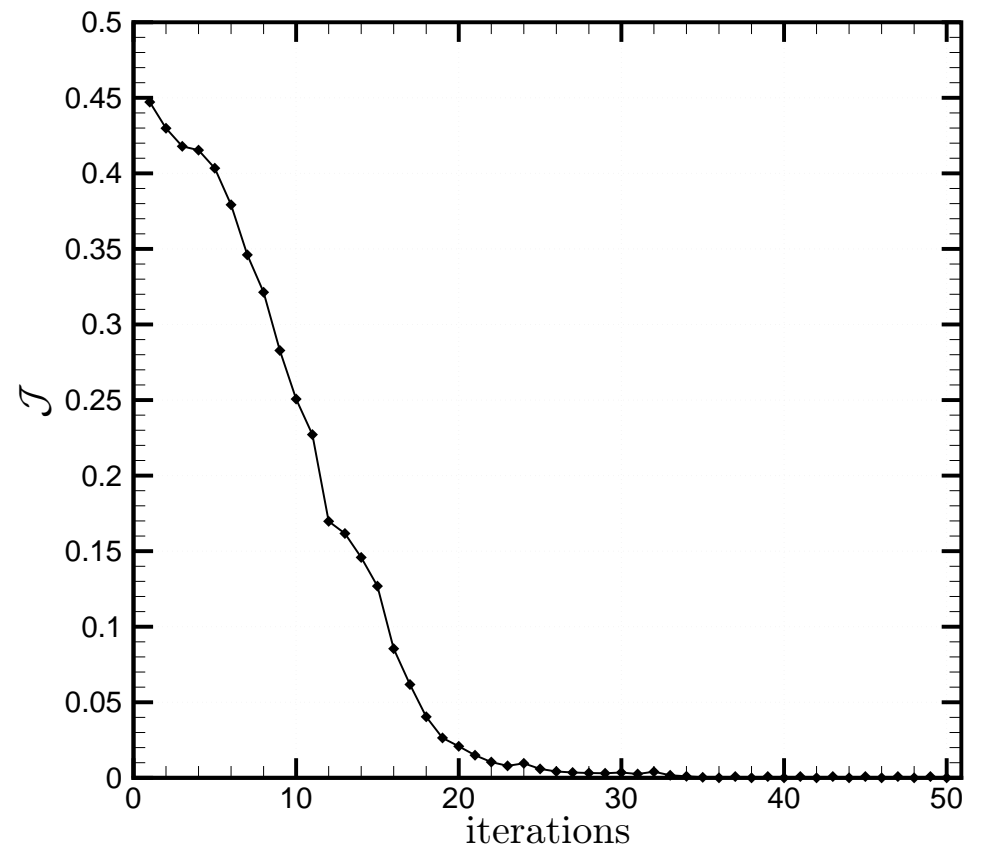

Figure 4: Variation of the functional as a function of the number of iterations. 


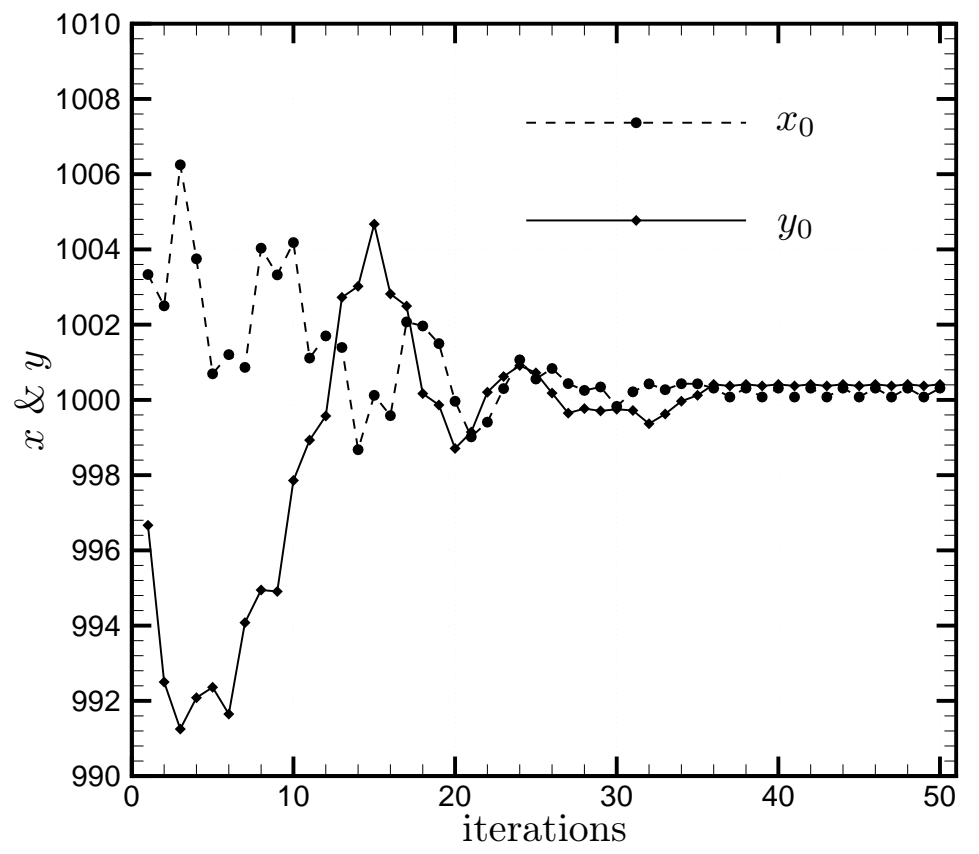

Figure 5: Variation of initial positions versus the number of iteration. 


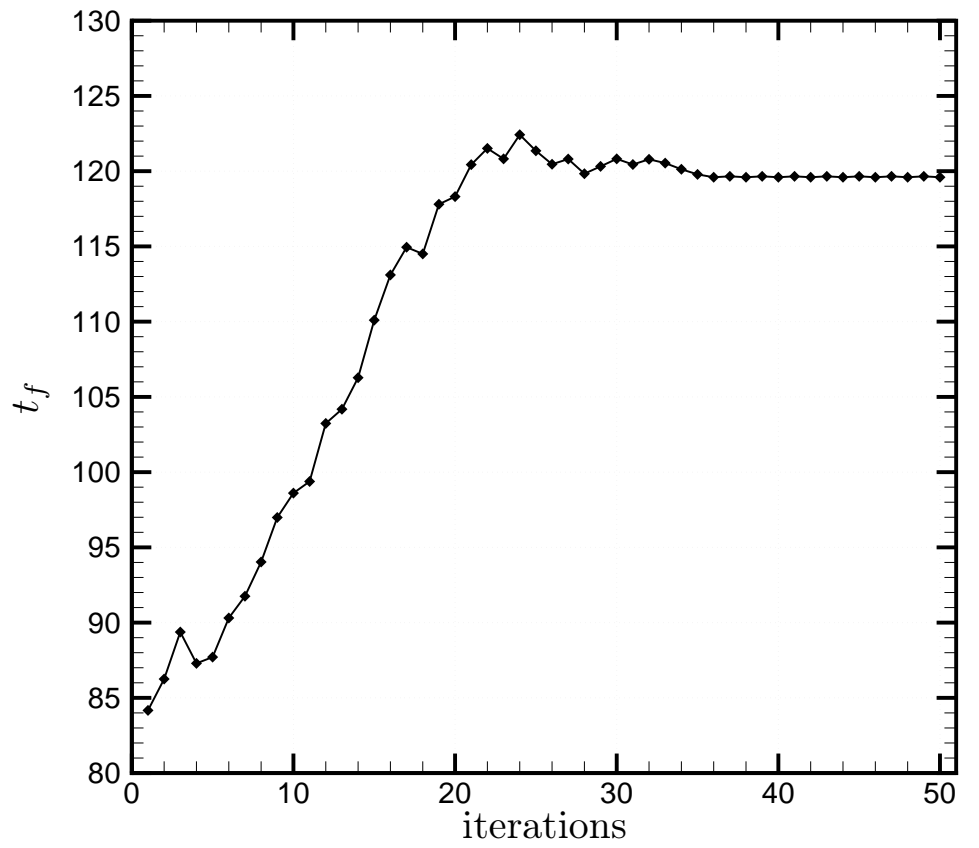

Figure 6: Variation of duration time versus the number of iteration. 


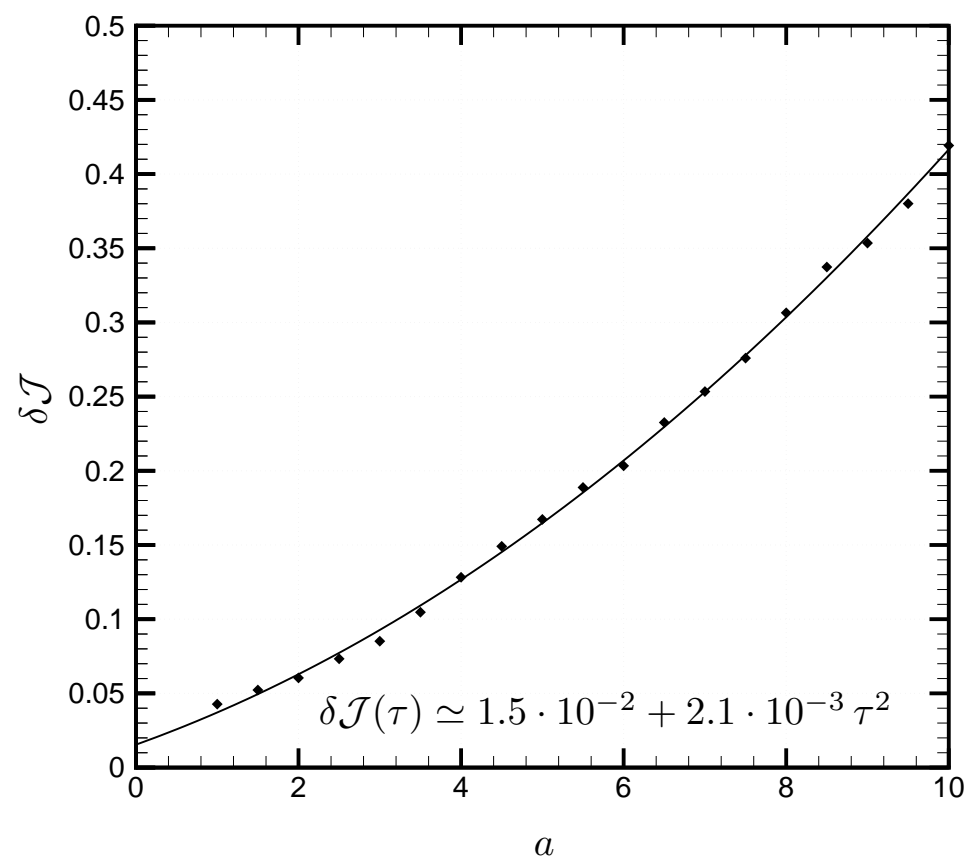

Figure 7: Sensibility of the objective function.

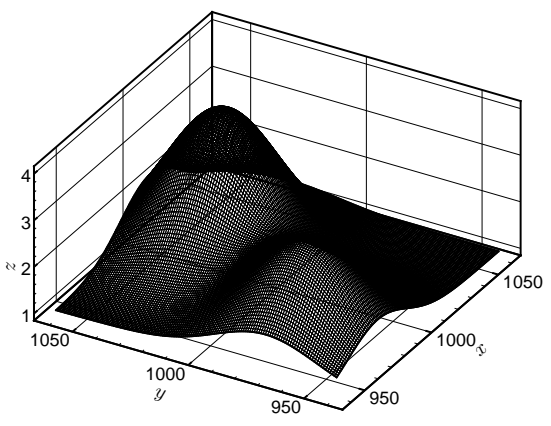

(a) $3 \mathrm{D}$ visualisation.

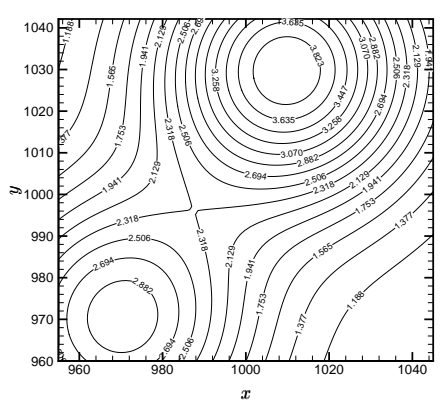

(b) $2 \mathrm{D}$ visualisation.

Figure 8: Topology of the non-uniform terrain. 


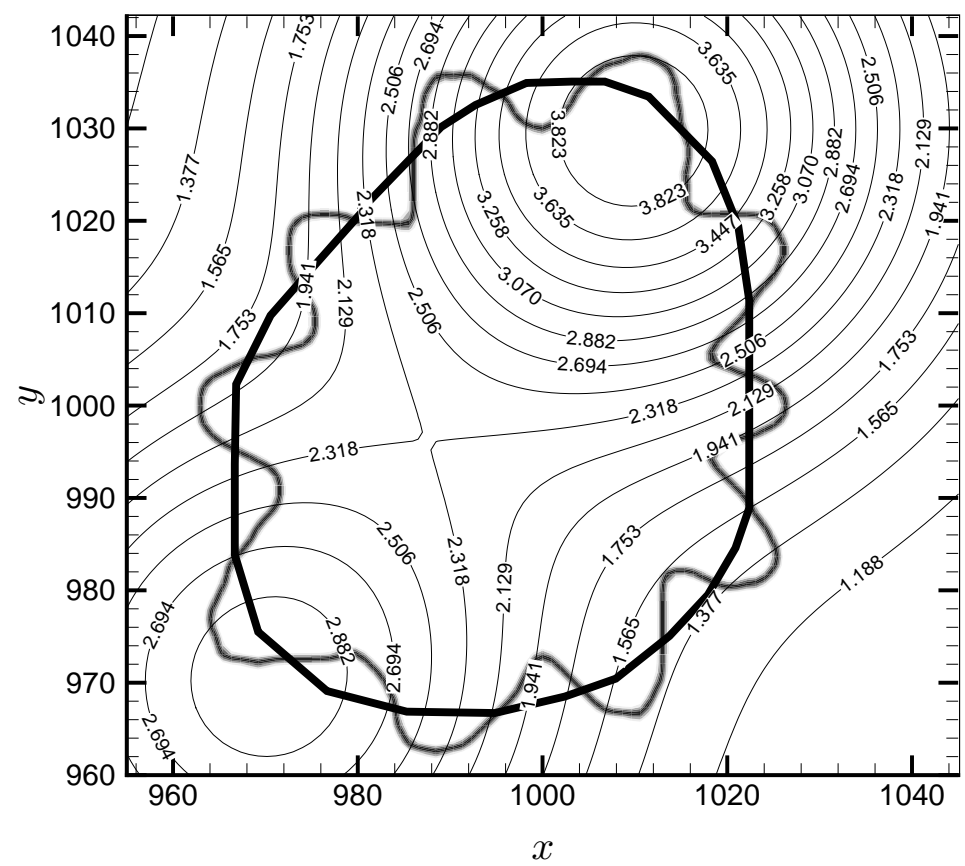

Figure 9: Real and perturbated fire front. Non-uniform terrain. 


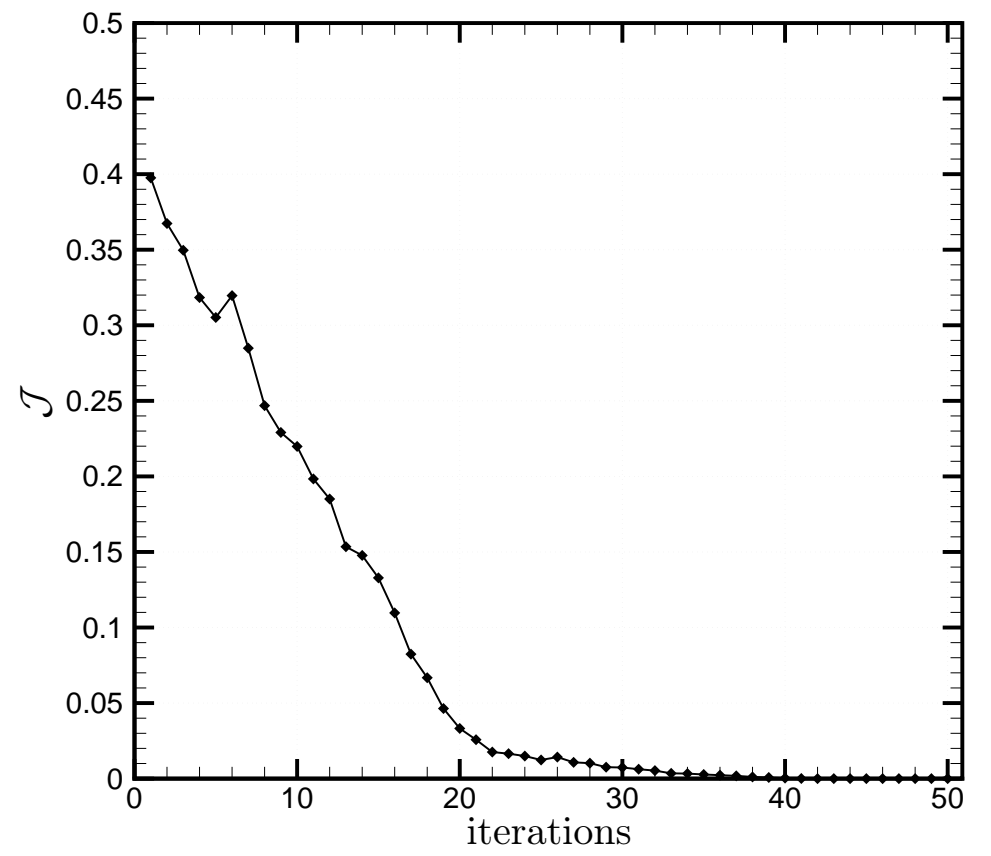

Figure 10: of the functional as a function of the number of iterations. Nonuniform terrain. 


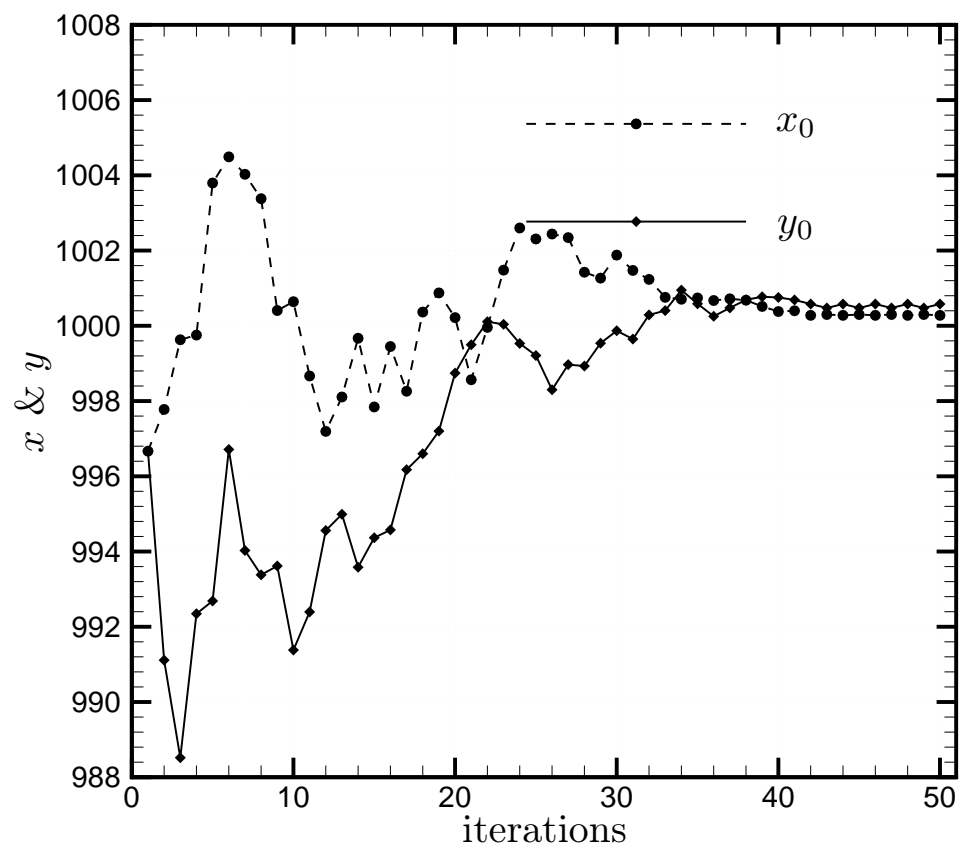

Figure 11: Variation of initial positions versus the number of iteration. Nonuniform terrain. 


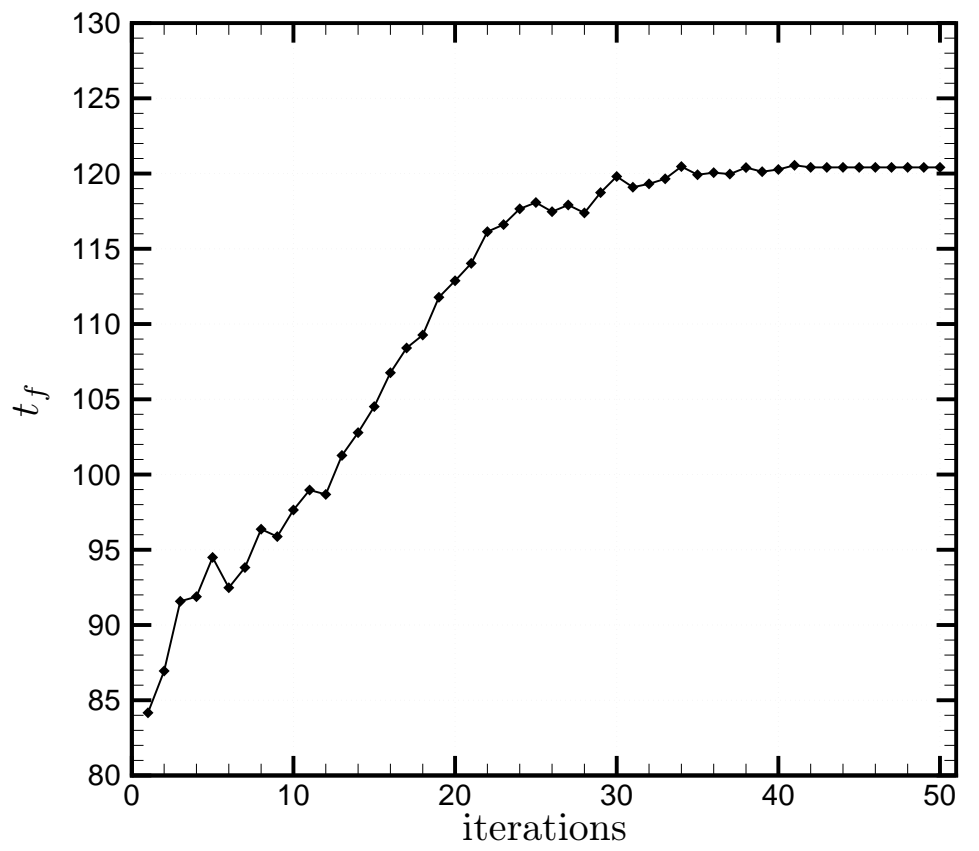

Figure 12: Variation of final time versus the number of iteration. Non-uniform terrain.

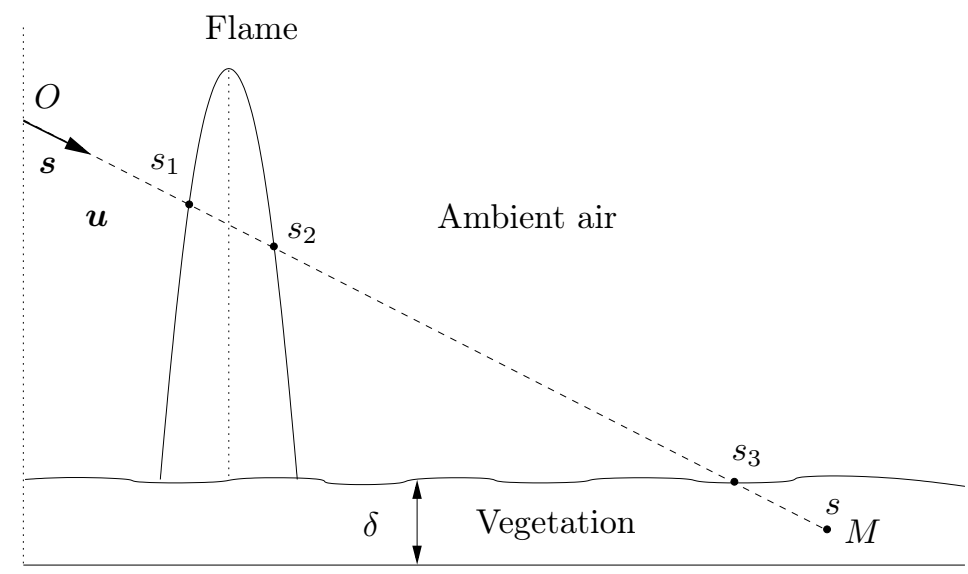

Figure 13: Configuration of the flame. 


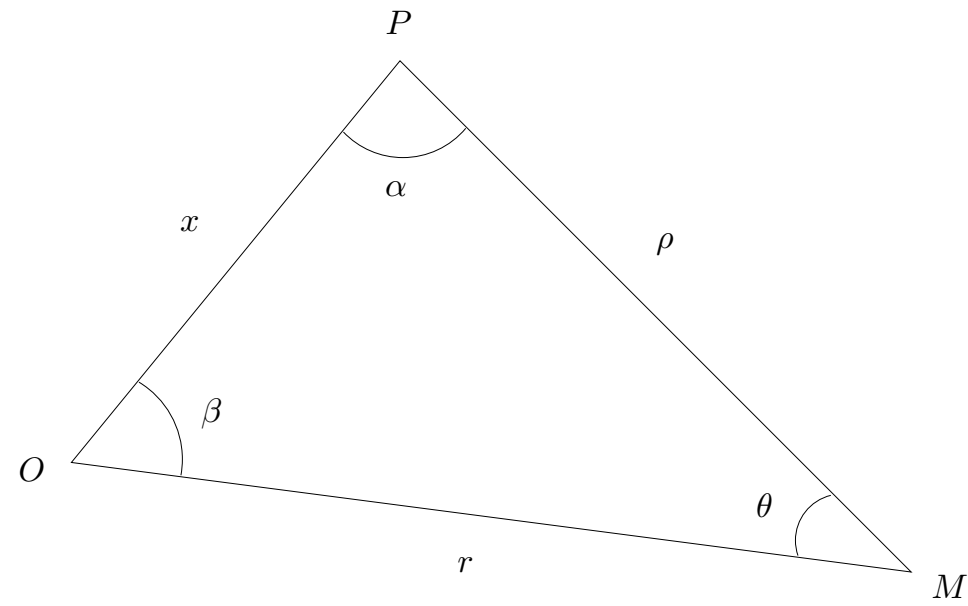

Figure 14: Définition of the different angles in the triangle $O P M$. 